\title{
Correlation between Chemical Composition and Antifungal Activity of Clausena lansium Essential Oil against Candida spp.
}

\author{
Xiaowen $\mathrm{He}^{1,2, \dagger}{ }^{1}$, Lantong Zhang ${ }^{2,+}$, Jinping Chen ${ }^{1}$, Jinlei Sui ${ }^{1}$, Guohui $\mathrm{Yi}^{1}{ }^{1}$, Jinyan $\mathrm{Wu}^{1}$ and \\ Yinzheng $\mathrm{Ma}^{3, *}$ \\ 1 Public Research Laboratory, Hainan Medical University, Haikou 571199, Hainan, China; \\ xiaowen_he@126.com (X.H.); 15595767678@163.com (J.C.); sui3com@126.com (J.S.); \\ guohuiyi6@163.com (G.Y.); jinyanzi0720@126.com (J.W.) \\ 2 School of Pharmacy, Hebei Medical University, Shijiazhuang 050017, Hebei, China; zhanglantong@263.net \\ 3 School of Public Health, Hainan Medical University, Haikou 571199, Hainan, China \\ * Correspondence: hbykdxmyz@163.com; Tel.: +86-898-6689-2235 \\ + These authors contributed equally to this work.
}

Academic Editors: Francesca Mancianti and Derek McPhee

Received: 7 March 2019; Accepted: 6 April 2019; Published: 9 April 2019

\begin{abstract}
Essential oils (EOs) have been shown to have a diversity of beneficial human health effects. Clausena is a large and highly diverse genus of plants with medicinal and cosmetic significance. The aim of this study was to analyze the composition of Clausena lansium EOs and to investigate their potential antifungal effects. The chemical compositions of Clausena lansium EOs obtained by hydrodistillation were analyzed by gas chromatography-mass spectrometry (GC-MS). A total of 101 compounds were identified among the diverse extracts of C. lansium. EOs of leaves and pericarps from different cultivars (Hainan local wampee and chicken heart wampee) collected in Hainan (China) were classified into four clusters based on their compositions. These clusters showed different antifungal activities against five Candida species (C. albicans, C. tropicalis, C. glabrata, C. krusei and C. parapsilosis) using the disc diffusion method. Clausena lansium EOs of pericarps displayed noteworthy antifungal activitives against all the tested Candida strains with inhibition zone diameters in the range of 11.1-23.1 mm. EOs of leaves showed relatively low antifungal activities with inhibition zone diameters in the range of $6.5-22.2 \mathrm{~mm}$. The rank order of antifungal activities among the four EO clusters was as follows: Cluster IV $>$ Cluster III $>$ Cluster I $\geq$ Cluster II. These results represent the first report about the correlation between chemical composition of $C$. lansium EOs and antifungal activity. Higher contents of $\beta$-phellandrene, $\beta$-sesquiphellandrene and $\beta$-bisabolene in EOs of pericarps were likely responsible for the high antifungal activity of Cluster IV EOs. Taken together, our results demonstrate the chemical diversity of Clausena lansium EOs and their potential as novel antifungal agents for candidiasis caused by Candida spp. Furthermore, the obtained results showing a wide spectrum of antifungal activities provide scientific evidence for the traditional use of these plants.
\end{abstract}

Keywords: correlation; Clausena lansium; essential oil; antifungal activity; Candida spp.

\section{Introduction}

Natural plant products, especially essential oils (EOs), have many beneficial biological effects, such as antibacterial, anti-inflammatory, antitumor and analgesic activities. EOs are volatile aromatic substances found in many plants. The EOs may exist in fruits, seeds, flowers and leaves. They have already significant attention because of their abundance, broad spectrum activities, and diverse mechanisms of action. They are among the most popular natural antimicrobial agents and they have recently gained a great popularity and scientific interest [1-4]. 
Clausena lansium (Lour.) Skeels, commonly known as wampee, is a tropical species that belongs to the family Rutaceae. It is reported as a native of Southern China, mainly distributed in Hainan, Fujian, Guangxi, Guangdong, Taiwan, etc. [5]. Clausena lansium has attracted great attention owing to its extensive pharmacological benefits, including anticancer, antioxidant, antidiabetic, antinociceptive, hepatoprotective, acerebroprotective and anti-trichomonal effects [6-9]. EOs of C. lansium play an important role in the abovementioned benefits [5]. In traditional Chinese medicine, the leaf and pericarp of C. lansium are often used to treat different diseases [10]. For example, the leaves of C. lansium have been used for treating fever, cough, asthma, malaria, dermatological and rheumatism, etc. When fruit turns from pale green to pale yellow or brownish-yellow around July, it can be eaten along with pericarp. Mature fruits have been used for treating digestive disorders, gastro-intestinal diseases and bronchitis. A likely mechanism is that EOs from C. lansium fruits can help release gastrointestinal gas, eliminates stagnation, dissipates heat and relieves pain.

Hainan Province, a tropical region of China, is one of the main geographic regions for growing Clausena lansium. Clausena lansium with globose fruits is named Hainan local wampee (HLW): the berry fruit is approximately $1.5-2.5 \mathrm{~cm}$ in diameter. Clausena lansium with broadly ovoid fruits is named chicken heart wampee (CHW), with berries approximately $1.5-2.5 \times 2.5-3.5 \mathrm{~cm}$ in diameter [11]. The compositions and contents of $C$. lansium EOs from China, Thailand, and Cuba have been studied by hydrodistillation followed by gas chromatography-mass spectrometry (GC-MS) [12-14]. However, limited effort has been made to analyze and compare the differences of EOs of HLW and CHW. In addition, due to the increasing number of fungal diseases caused by Candida species and insufficient effectiveness of traditionally applied azole agents, new therapeutic strategies are necessary and natural products especially EOs can play an important role in the treatment of infections $[15,16]$. Therefore, the search of new and effective natural antifungal agents has dramatically increased [17]. The aim of the current study was to comparatively analyze the chemical compositions of leaves and pericarps EOs of Clausena lansium of HLW and CHW and to investigate the in vitro antifungal activities against Candida species, including C. albicans, C. glabrata, C. krusei, C. parapsilosis and C. tropicalis. The results of this study can provide experimental basis for further exploitation of C. lansium.

\section{Results and Discussion}

\subsection{Extraction Results and Chemical Composition of EOs}

Clausena lansium EOs extracted by hydrodistillation were light yellow to yellow in color. The yields ranged from 0.23 to $0.51 \%(v / w)$. The source, cultivar, part, color and yield of EO details are listed in Table 1. There were differences in the color and yield of the eight EOs, which may be related to the geographic source, cultivar, and part of plants, but it is uncertain which is the most important of these factors. It is possible to come to a conclusion from the specific differences of the components. To compare the compositional differences, each EO extract was injected into GC-MS for analysis. Figure 1 shows GC-MS chromatograms of C. lansium EOs. The chemical compositions and contents results are shown in Table 2.

Table 1. Sources of Clausena lansium samples and extraction results of EOs.

\begin{tabular}{cccccc}
\hline No. & Source & Cultivar & Part & Color & Yield ${ }^{\text {a }}(\%, \boldsymbol{v} / \mathbf{w})$ \\
\hline MCL & Meixiao Village, Haikou City & CHW & Leaf & yellow & $0.23 \pm 0.08$ \\
MCP & Meixiao Village, Haikou City & CHW & Pericarp & light yellow & $0.48 \pm 0.10$ \\
RCL & Rulin Village, Haikou City & CHW & Leaf & yellow & $0.37 \pm 0.04$ \\
RCP & Rulin Village, Haikou City & CHW & Pericarp & light yellow & $0.37 \pm 0.09$ \\
MHL & Meixiao Village, Haikou City & HLW & Leaf & yellow & $0.47 \pm 0.08$ \\
MHP & Meixiao Village, Haikou City & HLW & Pericarp & light yellow & $0.50 \pm 0.07$ \\
RHL & Rulin Village, Haikou City & HLW & Leaf & yellow & $0.51 \pm 0.04$ \\
RHP & Rulin Village, Haikou City & HLW & Pericarp & light yellow & $0.47 \pm 0.05$ \\
\hline
\end{tabular}

${ }^{a}$ Values represent the means of three independent replicates $\pm \mathrm{SD}$. 


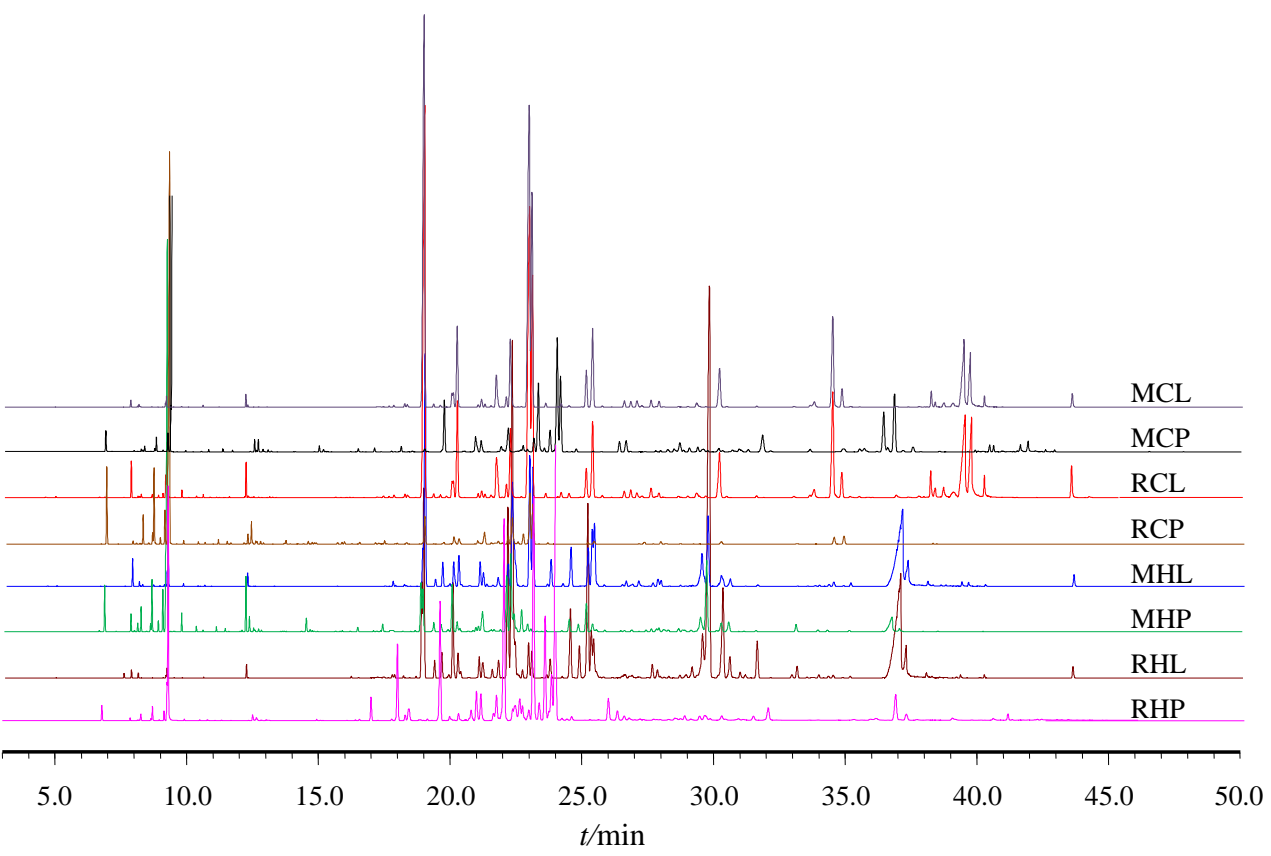

Figure 1. GC-MS chromatograms of Clausena lansium EOs.

Table 2. Chemical components and contents (\%) of Clausena lansium EOs.

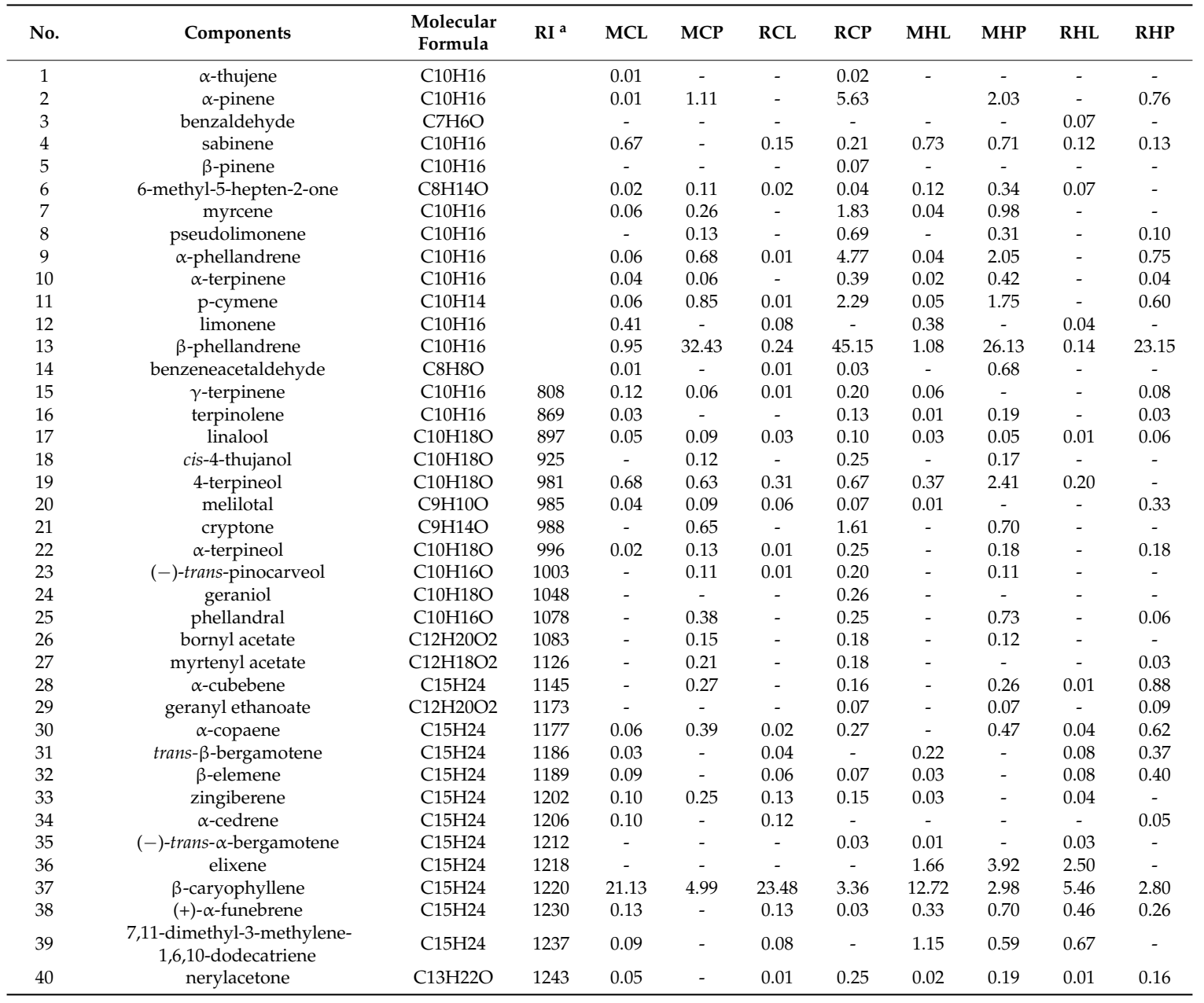


Table 2. Cont.

\begin{tabular}{|c|c|c|c|c|c|c|c|c|c|c|c|}
\hline No. & Components & $\begin{array}{l}\text { Molecular } \\
\text { Formula }\end{array}$ & $\mathbf{R I}^{\mathrm{a}}$ & MCL & МСР & RCL & RCP & MHL & MHP & RHL & RHP \\
\hline 41 & $\beta$-santalene & $\mathrm{C} 15 \mathrm{H} 24$ & 1244 & - & - & - & - & 0.14 & - & 0.11 & - \\
\hline 42 & (E)- $\beta$-farnesene & $\mathrm{C} 15 \mathrm{H} 24$ & 1249 & 1.12 & 1.86 & 1.15 & 1.00 & 1.37 & 3.60 & 2.62 & 4.04 \\
\hline 44 & $\alpha$-himachalene & $\mathrm{C} 15 \mathrm{H} 24$ & 1256 & - & 1.26 & - & 0.75 & 0.08 & 0.26 & 0.19 & 0.60 \\
\hline 45 & $(-)-\beta$-cadinene & $\mathrm{C} 15 \mathrm{H} 24$ & 1270 & - & - & - & - & - & 0.08 & - & - \\
\hline 46 & $\gamma$-muurolene & $\mathrm{C} 15 \mathrm{H} 24$ & 1275 & 0.13 & 0.57 & 0.11 & 0.37 & - & 0.34 & 0.01 & 0.32 \\
\hline 49 & (Z)- $\beta$-farnesene & $\mathrm{C} 15 \mathrm{H} 24$ & 1281 & 0.13 & - & 0.13 & - & 0.11 & - & 0.02 & - \\
\hline 50 & $\beta$-selinene & $\mathrm{C} 15 \mathrm{H} 24$ & 1287 & 0.13 & 0.19 & 0.07 & 0.11 & 0.06 & 0.13 & 0.26 & 0.21 \\
\hline 51 & $\gamma$-cadinene & $\mathrm{C} 15 \mathrm{H} 24$ & 1293 & 2.25 & 0.72 & 1.84 & 0.27 & 0.57 & 0.14 & - & 0.76 \\
\hline 52 & $\gamma$-elemene & $\mathrm{C} 15 \mathrm{H} 24$ & 1294 & - & 0.29 & - & 0.14 & - & 0.14 & 0.57 & 0.67 \\
\hline 53 & $\alpha$-muurolene & $\mathrm{C} 15 \mathrm{H} 24$ & 1297 & 0.02 & - & - & - & 0.02 & - & - & 0.84 \\
\hline 54 & $\alpha$-farnesene & $\mathrm{C} 15 \mathrm{H} 24$ & 1303 & 0.54 & 1.44 & 0.48 & 1.07 & $\begin{array}{l}0.02 \\
1.25\end{array}$ & 4.92 & 5.60 & 5.41 \\
\hline 55 & $\beta$-bisabolene & $\mathrm{C} 15 \mathrm{H} 24$ & 1306 & 2.99 & 5.61 & 3.46 & 3.88 & 5.30 & 7.74 & 9.08 & 10.21 \\
\hline 60 & $\alpha$-bergamotene & $\mathrm{C} 15 \mathrm{H} 24$ & 1325 & 9.49 & 7.09 & 10.63 & 5.41 & 1.88 & 0.23 & 0.83 & 1.02 \\
\hline 61 & cubinene & $\mathrm{C} 15 \mathrm{H} 24$ & 1330 & - & - & - & 0.03 & $\begin{array}{l}1.00 \\
-\end{array}$ & - & - & 0.39 \\
\hline 62 & $\alpha$-bisabolene & $\mathrm{C} 15 \mathrm{H} 24$ & 1336 & 0.16 & 0.31 & 0.17 & 0.14 & 0.13 & - & 0.09 & 0.27 \\
\hline 63 & acoradien & $\mathrm{C} 15 \mathrm{H} 24$ & 1340 & - & - & - & 0.02 & 1.55 & 0.09 & 0.58 & 0.68 \\
\hline 64 & alloaromadendrene oxide-(1) & $\mathrm{C} 15 \mathrm{H} 24 \mathrm{O}$ & 1351 & 0.22 & - & 0.16 & -0. & 0.14 & 0.08 & 0.04 & - \\
\hline 65 & trans-nerolidol & $\mathrm{C} 15 \mathrm{H} 26 \mathrm{O}$ & 1357 & 0.17 & - & 0.07 & - & 2.39 & 1.08 & 2.33 & 0.05 \\
\hline 66 & dendrolasin & $\mathrm{C} 15 \mathrm{H} 22 \mathrm{O}$ & 1368 & - & - & - & - & - & 0.67 & 1.05 & 0.64 \\
\hline 67 & spathulenol & $\mathrm{C} 15 \mathrm{H} 24 \mathrm{O}$ & 1372 & 1.33 & 0.10 & 1.96 & 0.17 & 2.34 & 2.65 & 6.38 & 3.61 \\
\hline 68 & caryophyllene oxide & $\mathrm{C} 15 \mathrm{H} 24 \mathrm{O}$ & 1377 & 3.64 & - & 4.50 & - & 7.82 & 0.85 & 3.18 & 0.43 \\
\hline 69 & Aromadendrene oxide-(1) & $\mathrm{C} 15 \mathrm{H} 24 \mathrm{O}$ & 1380 & - & - & - & 0.42 & 0.11 & 0.21 & 0.06 & 0.09 \\
\hline 70 & humuleneepoxide II & $\mathrm{C} 15 \mathrm{H} 24 \mathrm{O}$ & 1405 & 0.30 & 0.12 & 0.34 & - & 0.33 & 0.09 & 0.20 & 0.10 \\
\hline 71 & (-)-spathulenol & $\mathrm{C} 15 \mathrm{H} 24 \mathrm{O}$ & 1413 & - & 0.32 & - & - & - & 0.15 & 0.08 & 0.03 \\
\hline 72 & cis-Z- $\alpha$-bisabolene epoxide & $\mathrm{C} 15 \mathrm{H} 24 \mathrm{O}$ & 1416 & 0.25 & 0.47 & 0.38 & 0.08 & 0.38 & 0.16 & 0.05 & 0.20 \\
\hline 81 & $\alpha$-bisabolol & $\mathrm{C} 15 \mathrm{H} 26 \mathrm{O}$ & 1486 & 2.67 & 0.75 & 2.68 & 0.42 & 1.28 & 1.09 & 4.19 & 1.60 \\
\hline 82 & $\alpha$-santalol & $\mathrm{C} 15 \mathrm{H} 24 \mathrm{O}$ & 1493 & 0.06 & 0.16 & 0.04 & - & 0.60 & 1.07 & 0.93 & 2.01 \\
\hline 83 & $(Z, E)$ - $\alpha$-farnesene & $\mathrm{C} 15 \mathrm{H} 24$ & 1542 & 0.07 & 0.67 & 0.04 & - & - & 0.64 & 0.53 & 0.11 \\
\hline 84 & $\begin{array}{c}\text { tricyclopentadeca-3,7-dien } \\
{[8.4 .0 .1(11,14)]}\end{array}$ & $\mathrm{C} 15 \mathrm{H} 22 \mathrm{O}$ & 1557 & 0.13 & 0.47 & 0.11 & - & - & - & 0.02 & 0.10 \\
\hline 85 & ledol & $\mathrm{C} 15 \mathrm{H} 26 \mathrm{O}$ & 1562 & 0.56 & 0.67 & 0.46 & - & 0.05 & 0.16 & 0.02 & - \\
\hline 86 & $\beta$-santalol & $\mathrm{C} 15 \mathrm{H} 24 \mathrm{O}$ & 1569 & - & - & - & - & 0.12 & 0.14 & 0.07 & - \\
\hline 87 & $\alpha$-sinensal & $\mathrm{C} 15 \mathrm{H} 22 \mathrm{O}$ & 1573 & 5.83 & - & 5.88 & - & 0.29 & - & 0.10 & - \\
\hline 88 & $1,3,6,10$-farnesatetraen-12-al & $\mathrm{C} 15 \mathrm{H} 22 \mathrm{O}$ & 1582 & 1.20 & - & 1.04 & - & 0.04 & 0.13 & 0.01 & - \\
\hline 89 & santalol & $\mathrm{C} 15 \mathrm{H} 24 \mathrm{O}$ & 1641 & - & 2.45 & - & 1.11 & 17.31 & 2.92 & 12.24 & 4.40 \\
\hline 90 & trans-Z- $\alpha$-bisabolene epoxide & $\mathrm{C} 15 \mathrm{H} 24 \mathrm{O}$ & 1644 & - & 5.78 & - & 1.29 & 2.70 & 0.34 & 1.68 & 1.50 \\
\hline 91 & phytone & $\mathrm{C} 18 \mathrm{H} 36 \mathrm{O}$ & 1650 & - & - & 0.02 & - & 0.02 & - & - & - \\
\hline 92 & trans- $\beta$-santalol & $\mathrm{C} 15 \mathrm{H} 24 \mathrm{O}$ & 1670 & 0.03 & - & 0.01 & - & - & - & 0.14 & - \\
\hline 93 & cedr-8-en-13-ol & $\mathrm{C} 15 \mathrm{H} 24 \mathrm{O}$ & 1669 & 0.91 & 0.54 & 0.66 & - & 0.05 & - & 0.04 & 0.12 \\
\hline 94 & lanceol & $\mathrm{C} 15 \mathrm{H} 24 \mathrm{O}$ & 1676 & 0.39 & 0.46 & 0.20 & 0.06 & 0.07 & - & 0.03 & 0.68 \\
\hline 95 & linolenyl alcohol & $\mathrm{C} 18 \mathrm{H} 32 \mathrm{O}$ & 1690 & 0.51 & 0.10 & 0.26 & - & 0.06 & - & - & - \\
\hline 96 & farnesyl acetone & $\mathrm{C} 18 \mathrm{H} 30 \mathrm{O}$ & 1706 & 0.57 & 0.69 & 0.31 & - & - & - & 0.07 & - \\
\hline 97 & methyl palmitate & $\mathrm{C} 17 \mathrm{H} 34 \mathrm{O} 2$ & 1725 & 7.07 & 0.93 & 5.36 & - & 0.18 & - & - & - \\
\hline 98 & isophytol & $\mathrm{C} 2 \mathrm{OH} 40 \mathrm{O}$ & 1745 & 5.20 & - & 3.55 & - & - & - & - & - \\
\hline 99 & $n$-hexadecanoic acid & $\mathrm{C} 16 \mathrm{H} 32 \mathrm{O} 2$ & 1763 & 0.73 & - & 0.39 & - & 0.07 & - & 0.08 & - \\
\hline 100 & methyl elaidate & $\mathrm{C} 19 \mathrm{H} 36 \mathrm{O} 2$ & 1770 & 0.04 & - & - & - & - & - & - & - \\
\hline \multirow[t]{4}{*}{101} & \multirow{2}{*}{\multicolumn{2}{|c|}{$\begin{array}{l}\text { phytol } \\
\text { Total peak number }\end{array}$}} & 1847 & 1.19 & - & 0.60 & - & 0.58 & - & 0.31 & - \\
\hline & & & & 83 & 76 & 80 & 72 & 84 & 81 & 88 & 78 \\
\hline & \multicolumn{2}{|c|}{ Total identified peak number } & & 69 & 63 & 65 & 63 & 70 & 71 & 69 & 66 \\
\hline & \multicolumn{2}{|c|}{ Total identified peak area percentage } & & 98.87 & 96.90 & 99.19 & 98.18 & 98.10 & 98.60 & 96.87 & 96.39 \\
\hline
\end{tabular}

a RI: calculated Retention Index -: Not detected.

According to the GC-MS analysis, components and contents were different in leaves and pericarps of HLW and CHW. A total of 69, 63, 65, 63, 70, 71, 69 and 66 compounds were identified in MCL, MCP, RCL, RCP, MHL, MHP, RHL and RHP, amounting to $98.87 \%, 96.90 \%, 99.19 \%, 98.18 \%, 98.10 \%, 98.60 \%$, $96.87 \%$ and $96.39 \%$ of the total components, respectively. These components were monoterpenes, sesquiterpenes, alcohols, esters, etc. Some samples such as MCL and RCL were dominated by sesquiterpenes, with $\beta$-caryophyllene and $\beta$-sesquiphellandrene as the main components. MHL and 
RHL were rich in sesquiterpenes with cis- $\alpha$-santalol and santalol being the most abundant species. There were a large number of monoterpenes in some samples, such as MCP, RCP, MHP and RHP. In all the combined samples, we detected 101 chemicals in the EO extracts. However, the distributions of the 101 chemicals differed widely among the extracts. To investigate the similarity and differences among these extracts in EO profiles, a cluster analysis was conducted using SPSS (version 17.0). The clustering result is shown in the Figure 2.

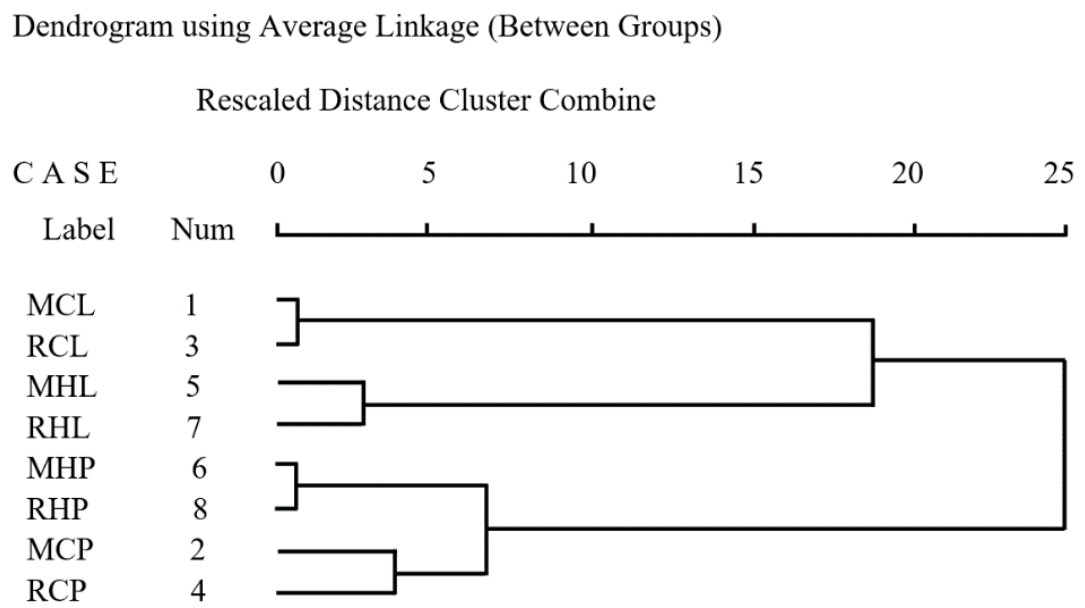

Figure 2. Dendrograms based on the chemical components and contents of Clausena lansium. Num: number.

Samples were clustered into two clusters for the first time, leaves and pericarps. This result indicates that most of the differences were caused by plant parts. The next contributor was plant cultivar, followed by geographic location of where the plants were grown. The difference between Meixiao Village and Rulin Village was the smallest, which is probably because the two places are geographically close (both in Haikou city). Table 3 shows the variation of some important volatile components (\%) of C. lansium in four clusters.

Table 3. Variation of some important volatile components (\%) of Clausena lansium in four clusters.

\begin{tabular}{ccccccccc}
\hline \multirow{2}{*}{ Components } & \multicolumn{2}{c}{ Cluster I } & \multicolumn{2}{c}{ Cluster II } & \multicolumn{2}{c}{ Cluster III } & \multicolumn{2}{c}{ Cluster IV } \\
\cline { 2 - 8 } & MCL & RCL & MHL & RHL & MHP & RHP & MCP & RCP \\
\hline 3-caryophyllene & $\mathbf{2 1 . 1 3}$ & $\mathbf{2 3 . 4 8}$ & 12.72 & 5.46 & 2.98 & 2.80 & 4.99 & 3.36 \\
$\beta$-sesquiphellandrene & $\mathbf{1 8 . 5 2}$ & $\mathbf{2 1 . 8 0}$ & 3.11 & 1.05 & 0.72 & 2.59 & $\mathbf{1 0 . 8 9}$ & $\mathbf{7 . 3 5}$ \\
cis- $\alpha$-santalol & 0.08 & 0.03 & $\mathbf{1 4 . 7 2}$ & $\mathbf{2 1 . 3 1}$ & 7.67 & 6.59 & 0.30 & 0.00 \\
$\beta$-santalol & 0.00 & 0.00 & $\mathbf{1 7 . 3 1}$ & $\mathbf{1 2 . 2 4}$ & 2.92 & 4.40 & 2.45 & 1.11 \\
$\beta$-phellandrene & 0.95 & 0.24 & 1.08 & 0.14 & $\mathbf{2 6 . 1 3}$ & $\mathbf{2 3 . 1 5}$ & $\mathbf{3 2 . 4 3}$ & $\mathbf{4 5 . 1 5}$ \\
$\beta$-bisabolene & 2.99 & 3.46 & 5.30 & 9.08 & $\mathbf{7 . 7 4}$ & $\mathbf{1 0 . 2 1}$ & 5.61 & 3.88 \\
$\alpha$-bergamotene & 9.49 & 10.63 & 1.88 & 0.83 & 0.23 & 1.02 & 7.09 & 5.41 \\
\hline
\end{tabular}

The domonant EOs in each of the four clusters are listed below:

Cluster I: $\beta$-caryophyllene and $\beta$-sesquiphellandrene. MCL and RCL belonged to this cluster. These samples were leaves of CHW. The components were rich in $\beta$-caryophyllene $(21.13 \%, 23.48 \%)$, $\beta$-sesquiphellandrene $(18.52 \%, 21.80 \%)$, and accompanied by $\alpha$-bergamotene $(9.49 \%, 10.63 \%)$.

Cluster II: cis- $\alpha$-santalol and $\beta$-santalol. There were MHL and RHL in this cluster. These samples were leaves of HLW. cis- $\alpha$-Santalol (14.72\%, 21.31\%), $\beta$-santalol (17.31\%, 12.24\%) and caryophyllene $(12.72 \%, 5.46 \%)$ were the most predominant components.

Cluster III: $\beta$-phellandrene and $\beta$-bisabolene. MHP and RHP were classified into this cluster. They were pericarps of HLW. They contained more $\beta$-phellandrene, a relative percentage of $26.13 \%$ and $23.15 \%$, respectively. Percentages of $\beta$-bisabolene and cis- $\alpha$-santalol were also relatively large $(6.59-10.21 \%)$. 
Cluster IV: $\beta$-phellandrene and $\beta$-sesquiphellandrene. Pericarps of CHW such as MCP and RCP in this cluster. $\beta$-Phellandrene in this cluster had a percentage higher than $30 \%$. The percentages of $\beta$-phellandrene were $32.43 \%$ and $45.15 \%$ in MCP and RCP, respectively. $\beta$-Sesquiphellandrene was also relative high, with percentages of $10.89 \%$ and $7.35 \%$, respectively.

In previous studies, EOs of C. lansium from China [12], Thailand [13], and Cuba [14] were obtained by hydrodistillation and analyzed by GC-MS. Thirty-two components were identified in leaves samples from China. The main components identified in the EO of leaves were $\beta$-santalol (35.20\%) and bisabolol $(13.70 \%)$. In both fresh and dried fruit samples from Thailand, fifty-three components were identified, and the main components were sabinene (33.68-66.73\%), $\alpha$-pinene (9.57-13.35\%) and 1-phellandrene (5.77-10.76\%). For leaf samples from Cuba, seventy compounds were identified. The most prominent components were caryophyllene oxide (16.80\%) and (Z)- $\alpha$-santalol $(11.70 \%)$. The components and percentages were different from different areas, and the differences were much greater in different parts of C. lansium. There were some common components in EOs of leaves in the present study and the above reports, such as cis- $\alpha$-santalol, $\beta$-bisabolene, and caryophyllene oxide. In contrast, the components of pericarps in our study were very different from those of the fruit samples in previous studies. The reason may be that fruit contains not only pericarp, but also pulp and seed.

In addition to the hydrodistillation method, supercritical fluid extraction (SFE) was used in the extraction of C. lansium leaves from Guangdong, China [18]. Thirty-six components were identified and the main components were 4-terpineol (26.94\%) and $\gamma$-terpinene (14.39\%). Chokeprasert et al. [19] analyzed the volatile components of fresh leaves and pericarps in Thailand by headspce (HS) GC-MS. Thirty-nine components were identified in the leaves, and the major components were sabinene $(15 \%)$ and $\beta$-bisabolene (9.88\%); thirty components were identified in the pericarps, and the major components were sabinene $(69.07 \%)$ and $\alpha$-phellandrene $(10.63 \%)$. The components and percentages obtained by SFE and HS were quite different from that by hydrodistillation.

In the present study, the differences of the compositions of Clausena lansium leaves and pericarps from different cultivars were analyzed and compared for the first time. Cluster analysis result showed that the differences in the components were most significantly contributed by plant parts, followed by cultivar, and with geographic origin contributing the least to the variation.

\subsection{Antifungal Activity}

The antifungal activity of C. lansium EOs was examined using the filter paper disc diffusion method. As shown in Table 4, the EOs inhibited the growth of all seven yeast strains tested in our study. The inhibition zone diameters were in the range of 6.5-23.1 mm. The EOs of Clausena lansium pericarps showed significant activity against all the Candida strains, whereas, EOs of leaves showed relatively poor activity. The RCP EO exhibited the greatest antifungal effect against C. glabrata, with an inhibition zone diameter of $23.1 \mathrm{~mm}$. Notably, C. albicans 27, a yeast strain which is resistant to FLZ and AMB, can be inhibited by Clausena lansium EOs with an inhibition zone diameter of 11.3-15.3 mm. This observation suggests that Clausena lansium EOs is active towards certain FLZ and AMB resistant strains.

Table 4. Antifungal activity of Clausena lansium EOs $(x \pm s)$.

\begin{tabular}{|c|c|c|c|c|c|c|c|c|c|c|}
\hline \multirow{2}{*}{ Candida Strains } & \multicolumn{10}{|c|}{ Zone of Inhibition $(\mathrm{mm})^{a}$} \\
\hline & MCL & RCL & MHL & RHL & MHP & RHP & МCP & $\mathrm{RCP}$ & FLZ & AMB \\
\hline C. albicans ATCC 10231 & $8.0 \pm 0.2$ & $9.0 \pm 0.2$ & $8.0 \pm 0.2$ & $8.8 \pm 0.3$ & $12.8 \pm 0.3$ & $12.5 \pm 0.2$ & $13.5 \pm 0.3$ & $13.2 \pm 0.3$ & $33.2 \pm 0.4$ & $23.6 \pm 0.1$ \\
\hline C. tropicalis $\mathrm{CMCC}(\mathrm{F}) \mathrm{c} 2 \mathrm{f}$ & $7.6 \pm 0.2$ & $6.5 \pm 0.2$ & $7.5 \pm 0.1$ & $6.5 \pm 0.1$ & $15.0 \pm 0.1$ & $15.5 \pm 0.3$ & $12.2 \pm 0.3$ & $12.4 \pm 0.2$ & $35.8 \pm 0.4$ & $23.6 \pm 0.3$ \\
\hline C. parapsilosis ATCC 22019 & $11.0 \pm 0.2$ & $10.0 \pm 0.2$ & $8.4 \pm 0.2$ & $7.9 \pm 0.3$ & $11.1 \pm 0.2$ & $11.4 \pm 0.2$ & $12.1 \pm 0.2$ & $12.0 \pm 0.2$ & $32.4 \pm 0.4$ & $23.7 \pm 0.2$ \\
\hline C. glabrata $\mathrm{CMCC}(\mathrm{F})$ c6e & $22.0 \pm 0.3$ & $22.2 \pm 0.2$ & $20.0 \pm 0.3$ & $20.9 \pm 0.2$ & $22.1 \pm 0.3$ & $22.0 \pm 0.2$ & $22.8 \pm 0.3$ & $23.1 \pm 0.3$ & $20.2 \pm 0.2$ & $22.0 \pm 0.2$ \\
\hline C. albicans 53 & $8.2 \pm 0.2$ & $8.8 \pm 0.2$ & $8.1 \pm 0.1$ & $8.6 \pm 0.3$ & $12.9 \pm 0.2$ & $12.4 \pm 0.1$ & $14.5 \pm 0.3$ & $14.2 \pm 0.2$ & $32.2 \pm 0.3$ & $23.8 \pm 0.2$ \\
\hline C. albicans 27 & $13.0 \pm 0.2$ & $12.4 \pm 0.2$ & $11.5 \pm 0.2$ & $11.3 \pm 0.2$ & $14.2 \pm 0.3$ & $15.3 \pm 0.2$ & $14.0 \pm 0.2$ & $13.3 \pm 0.1$ & $11.0 \pm 0.2$ & $8.2 \pm 0.2$ \\
\hline
\end{tabular}


It can be seen from Table 4 , the antifungal activitives efficacy order was as follows: Cluster IV $\geq$ Cluster III > Cluster I $\geq$ Cluster II. The two most active EOs against C. albicans, C. glabrata, C. krusei and C. parapsilosis, which were pericarps of CHW (Cluster IV) and were all rich in $\beta$-phellandrene (32.43\%, $45.15 \%)$ and $\beta$-sesquiphellandrene $(10.89 \%, 7.35 \%)$. Inhibition zone diameters were in the range of 12.0-23.1 mm. EOs of pericarps of HLW (Cluster III) were the most active against C. tropicalis and C. albicans 27 (a clinical strains resistant to FLZ and AMB), with the most predominant components being $\beta$-phellandrene $(26.13 \%, 23.15 \%)$ and $\beta$-bisabolene $(7.74 \%, 10.21 \%)$. Inhibition zone diameters were in the range of $11.1-22.1 \mathrm{~mm}$. The percentages of the monoterpene $\beta$-phellandrene in the two clusters were all high. There were some differences of compositions between Cluster I and II, but they were all dominated by sesquiterpenes as the main components. The antifungal activities against C. glabrata were almost equal to Cluster IVand III with inhibition zone diameters 22.0-22.2 mm. However, the antifungal activities against other six Candida strains were relatively low, with inhibition zone diameters 6.5-13.0 mm. Antifungal effect of EOs of CHW was higher than that of HLW in both leaves and pericarps. Comprehensive analysis illustrates the importance of monoterpenes to antifungal activities and there were differences among different types of Candida strains. The results agree with those of Białon et al. [20] who suggested that the antifungal potential of lemon essential oils against Candida yeast strains was related to the high content of monoterpenoids and the type of Candida strains.

There are few studies reporting the antifungal activity of Clausena lansium extracts against infectious yeasts. XU et al. [21] reported that the extracts of pericarps of Clausena lansium using 95\% alcohols had the significant inhibitory effect on C. albicans with an inhibition zone diameter of $16.8 \mathrm{~mm}$ by the filter paper disc diffusion method. However, the components of this extracts are not yet clear. In the present study, we obtained Clausena lansium EOs by hydrodistillation and determined the chemical compositions by GC-MS. In all the tested EOs of pericarps, $\beta$-phellandrene was determined to be present at the highest percentage which could be mainly responsible for the antifungal effects. $\beta$-Sesquiphellandrene and $\beta$-bisabolene were also important compositions which were possible to have antifungal activities. Perigo et al. [22] studied the chemical compositions and antibacterial activities of Piper species from distinct rainforest areas in Southeastern Brazil, and found that higher contents of $\beta$-phellandrene were positively correlated to wide spectrum antibacterial activity. Antibacterial activity of essential oils of Tripleurospermum disciforme was reported that the reason for higher antibacterial effect is the presence of $\beta$-farnesene and $\beta$-sesquiphellandrene [23]. The essential oils of Bocageopsis pleiosperma Maas rich in $\beta$-bisabolene were reported to have high antifungal activities [24]. Their results are consistent with ours. Taken together, we speculate that antifungal activities in our study may be attributed to C. lansium EOs of pericarps rich in $\beta$-phellandrene, $\beta$-sesquiphellandrene and $\beta$-bisabolene.

However, antifungal activity may not entirely depend on the main chemical components. Other chemical components in lower concentrations may also have antifungal activity or have synergistic effects with other components. Therefore, it is necessary to study antifungal activity of the component alone or in combination with others, in order to further elucidate the material basis of antifungal activity of $C$. lansium EOs against Candida spp. It may provide a basis for the development of C. lansium EOs as new antifungal agents with high efficiency, broad spectrum, low toxicity and low cost.

\section{Conclusions}

This study described the chemical compositions of the EOs from leaves and pericarps of Clausena lansium (Lour.) Skeels, where a total 101 compounds were identified. EOs of leaves and pericarps of HLW and CHW in Hainan, China were classified into four clusters by cluster analysis. The major components of cluster I were caryophyllene $(21.13 \%, 23.48 \%)$, $\beta$-sesquiphellandrene $(18.52 \%$, $21.80 \%)$ and $\alpha$-bergamotene $(9.49 \%, 10.63 \%)$; Cluster II: cis- $\alpha$-santalol $(14.72 \%, 21.31 \%)$, santalol $(17.31 \%, 12.24 \%)$ and caryophyllene (12.72\%, 5.46\%); Cluster III: $\beta$-phellandrene $(26.13 \%, 23.15 \%)$ and $\beta$-bisabolene $(7.74 \%, 10.21 \%)$; Cluster IV: $\beta$-phellandrene $(32.43 \%, 45.15 \%)$ and $\beta$-sesquiphellandrene 
$(10.89 \%, 7.35 \%)$. Correlation between chemical composition and antifungal activity is an important finding. EOs of Clausena lansium pericarps showed higher antifungal activity than that of leaves against all the tested Candida strains, and EOs of CHW had better inhibiting effect than HLW. Higher contents of $\beta$-phellandrene, $\beta$-sesquiphellandrene and $\beta$-bisabolene may be positively correlated to antifungal activity. The present results increase the biological knowledge about Clausena lansium EOs, which could be exploited particularly for the treatment and prevention of fungal infections caused by Candida spp.

\section{Materials and Methods}

\subsection{Samples and Chemicals}

The leaves and fruits of Clausena lansium were collected from Haikou City in Hainan, China, in June 2018. Figure 3 shows the leaves and fruits of HLW (a) and CHW (b). All samples were identified by Professor Jianping Tian and were deposited in Public Research Center, Hainan Medical University (Hainan, China). Table 1 lists the sources of C. lansium employed in this study. C8-C40 n-alkanes (500 mg. $\mathrm{L}^{-1}$ in hexane) were purchased from America o2si (Charleston, SC, USA). Other chemicals and their suppliers are listed as follows: agar powder (BeijingSolarbio Sci. \& Tech. Co., Ltd., Beijing, China), glucose (Tianjin Fu Chen Chemical Reagent Factory, Tianjin, China), tryptone (Guangdong Huankai Microbial Sci. \& Tech. Co., Ltd., Guangdong, China), MH agar powder (Guangdong Huankai Microbial Sci. \& Tech. Co., Ltd., Guangdong, China), fluconazole (FLZ) and amphotericin B (AMB) (Aladdin, Shanghai, China), n-hexane (chromatographic purity, Aladdin).

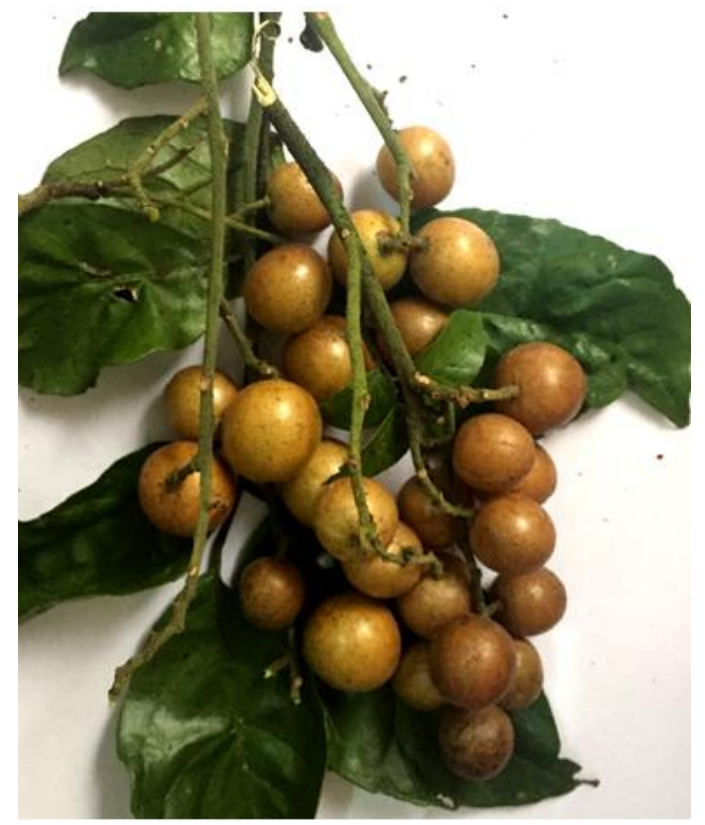

(a)

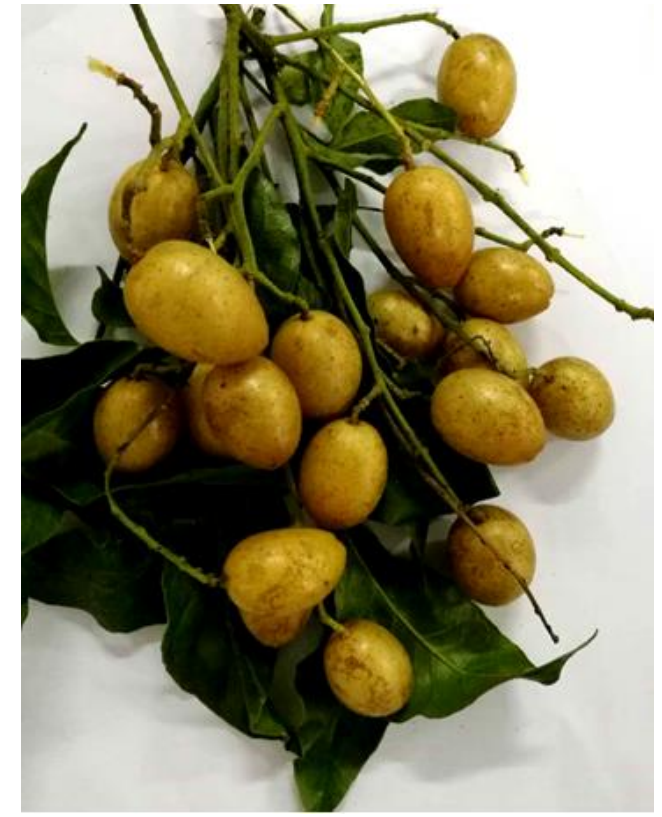

(b)

Figure 3. Leaves and ripe fruits of Hainan local wampee (HLW) (a) and chicken heart wampee (CHW) (b).

\subsection{Instrumentation and Methods}

\subsubsection{Instrumentation}

Measurements were carried out on a GC-MS-QP2010-Plus system (Shimadzu, Kyoto, Japan) in electron impact ionization (EI) mode. Data were processed with the Shimadzu GC-MS Solution software. The column was ZB-5MS fused silica (30 m $\times 2.5 \mathrm{~mm} ; 0.25 \mu \mathrm{m}$ film thickness) from Phenomenex, Torrance, CA, USA. Other instrumentation included a FW-80 high speed grinder (Tianjin Taisite Instrument Co., Ltd, Tianjin, China), an AL104 electronic balance (METTLER TOLEDO 
Instrument Co., Ltd., Shanghai, China), Automatic digital gel image analysis system Tanon-4100 (Shanghai Tianlong Technology Co., Ltd., Shanghai, China), SPX-250B-Z biochemical incubator (Shanghai Bo Xun Industrial Co., Ltd., Shanghai, China).

\subsubsection{Hydrodistillation}

The collected leaves of Clausena lansium were dried in shade. After removing pulps and seeds of fruits, pericarps were washed and then dried in an oven at $40-50{ }^{\circ} \mathrm{C}$. The leaves and pericarps were milled into powder with a grinder. The EOs were extracted by hydrodistillation for 2.5-3.0 $\mathrm{h}$ in a Clevenger-type apparatus. The obtained EOs were stored at $4{ }^{\circ} \mathrm{C}$ in an air-tight container and dried using anhydrous sodium sulfate before being analyzed.

\subsubsection{GC-MS Analysis}

Samples were analyzed by the GC-MS EI method with column ZB-5MS. Helium was used as a carrier gas at a flow rate of $1.0 \mathrm{~mL} \cdot \mathrm{min}^{-1}$ in split mode (1:20). The column temperature was maintained at $60{ }^{\circ} \mathrm{C}$ for $5 \mathrm{~min}$ and then programmed to $120^{\circ} \mathrm{C}$ at a heating rate of $10^{\circ} \mathrm{C} \cdot \mathrm{min}^{-1}$, further increased to $170^{\circ} \mathrm{C}$ at a rate of $2{ }^{\circ} \mathrm{C} \cdot \mathrm{min}^{-1}$, and finally increased to $210^{\circ} \mathrm{C}$ at a rate of $10^{\circ} \mathrm{C} \cdot \mathrm{min}^{-1}$ for remaining $10 \mathrm{~min}$. Temperatures of both injector and connector were maintained at $270^{\circ} \mathrm{C}$. Operating parameters of MS were: EI mode at $70 \mathrm{eV}$ with a mass scanning range of 50-500 amu and source temperature of $250{ }^{\circ} \mathrm{C}$. C $8-\mathrm{C} 40 n$-alkanes were used as reference points in the calculation of relative retention indexes (RIs). The percentages of compositions were obtained from the electronic integration of peak areas. The identity of each compound was determined by comparing its RI to $n$-alkanes and the NIST library database (NIST08 and NIST08s). Percentage of composition was computed from peak areas without applying correction factors.

\subsubsection{Candida Strains and Culture Media}

The Candida strains include the standard reference strains (C. albicans ATCC 10231, C. parapsilosis ATCC 22019, C. krusei ATCC 6258, C. tropicalis CMCC(F) c2f, and C. glabrata CMCC(F) c6e), and the clinical strains (C. albicans 53, C. albicans 27). The clinical strains were from oral mucosa of patients with oral candidiasis in the Hainan Wenchang General Hospital located in Wenchang, China. Two clinical isolates were previously identified based on ITS gene sequence analysis. The obtained yeast isolates were stored at $-80^{\circ} \mathrm{C}$ freezer until use. All strains were streaked onto plates containing the sabouraud dextrose agar (SDA) plate at $35^{\circ} \mathrm{C}$ for $24 \mathrm{~h}$. SDA plate composed of $2 \%(w / v)$ agar powder, $2 \%$ glucose, and $1 \%$ tryptone. A single colony was streaked again to ensure the viability and purity of the strains and the colonies were incubated at $35{ }^{\circ} \mathrm{C}$ for $24 \mathrm{~h}$. The colonies with the diameter of about $1 \mathrm{~mm}$ were selected and microbial suspensions were prepared in a saline solution. The $\mathrm{MH}$ agar plate was used for testing antifungal susceptibilities by the filter paper disc diffusion method.

\subsubsection{Probing the Antifungal Activity of the EO Sample by the Filter Paper Disc Diffusion Method}

The antifungal activity of the EOs of C. lansium was first investigated using the filter paper disc diffusion method following the protocol described in CLSI M44-A2 [25]. Microbial suspensions were prepared in a saline solution and standardized to a turbidity equivalent to that of the tube No. 0.5 on the McFarland scale, corresponding to approximately $1-5 \times 10^{6} \mathrm{CFU} \cdot \mathrm{mL}^{-1}$. The antifungal drug FLZ $\left(2.5 \mathrm{mg} \cdot \mathrm{mL}^{-1}\right)$ and AMB $\left(1.0 \mathrm{mg} \cdot \mathrm{mL}^{-1}\right)$, which are commonly used to treat candidiasis, were used as positive controls. $10 \mu \mathrm{L}$ of Clausena lansium EOs and positive controls was respectively added onto a piece of paper placed on the medium in a plate containing the fungal lawn. The plates were incubated at $35^{\circ} \mathrm{C}$ for 24 to $48 \mathrm{~h}$, respectively. The antifungal activity the samples was evaluated by measuring zones of inhibition of fungal growth surrounding the paper discs. The zones of inhibition were measured with antibiotic zone scale (Cecon-Brasil) in $\mathrm{mm}$. All the experiments were carried out in triplicate. When the inhibition zone diameters of FLZ and AMB against $\mathrm{Ca}$ ATCC 10231 were within 
the prescribed range 28-39 $\mathrm{mm}$ and 20-27 $\mathrm{mm}$, respectively, it is considered that the operation of this test was effective.

Author Contributions: Data curation, J.W.; Formal analysis, J.S.; Investigation, L.Z. and Y.M.; Methodology, X.H. and J.C.; Software, G.Y.; Validation, L.Z.; Writing—original draft, X.H., Writing—review \& editing, Y.M.

Funding: This research was supported by Scientific Research Projects of Higher Education Institutions of Hainan Province (Hnky2019-46); National Natural Science Foundation of China (31860035). We thank JianPing Xu from Department of Biology, McMaster University for revising the manuscript.

Conflicts of Interest: All the authors have declared no conflict of interest.

$\begin{array}{ll}\text { Abbreviations } \\ \text { EO } & \text { essential oil } \\ \text { GC-MS } & \text { gas chromatography-mass spectrometry } \\ \text { HLW } & \text { Hainan local wampee } \\ \text { CHW } & \text { chicken heart wampee } \\ \text { FLZ } & \text { fluconazole } \\ \text { AMB } & \text { amphotericin B } \\ \text { EI } & \text { electron impact ionization } \\ \text { RI } & \text { retention indexe } \\ \text { SDA } & \text { sabouraud dextrose agar } \\ \text { HS } & \text { headspace } \\ \text { SFE } & \text { supercritical fluid extraction }\end{array}$

\section{References}

1. Bakkali, F.; Averbeck, S.; Averbeck, D.; Idaomar, M. Biological effects of essential oils-A review. Food Chem. Toxicol. 2008, 2, 446-475. [CrossRef] [PubMed]

2. Qi, X.L.; Li, T.T.; Wei, Z.F.; Guo, N.; Luo, M.; Wang, W.; Zu, Y.G.; Fu, Y.J.; Peng, X. Solvent-free microwave extraction of essential oil from pigeon pea leaves (Cajanus cajan (L.) Millsp.) and evaluation of its antimicrobial activity. Ind. Crop. Prod. 2014, 58, 322-328. [CrossRef]

3. Dosoky, N.S.; Setzer, W.N. Biological Activities and Safety of Citrus spp. Essential Oils. Int. J. Mol. Sci. 2018, 19, 1966. [CrossRef] [PubMed]

4. Mezza, G.N.; Borgarello, A.V.; Grosso, N.R.; Fernandez, H.; Pramparo, M.C.; Gayol, M.F. Antioxidant activity of rosemary essential oil fractions obtained by molecular distillation and their effect on oxidative stability of sunflower oil. Food Chem. 2018, 242, 9-15. [CrossRef] [PubMed]

5. Lim, T.K. Clausena lansium. In Edible Medicinal And Non-Medicinal Plants, 1st ed.; Springer: Berlin, Germany, 2012; pp. 871-883.

6. Shen, D.Y.; Chao, C.; Chan, H.H.; Huang, G.J.; Hwang, T.L.; Lai, C.; Lee, K.H.; Thang, T.D.; Wu, T.S. Bioactive constituents of Clausena lansium and a method for discrimination of aldose enantiomers. Phytochem. 2012, 82, 110-117. [CrossRef] [PubMed]

7. Adebajo, A.C.; Iwalewa, E.O.; Obuotor, E.M.; Omisore, G.F.; Adewunmi, C.O.; Obaparusi, O.O.; Klaes, M.; Adetogun, G.E.; Schmidt, T.J.; Verspohl, E.J. Pharmacological properties of the extract and some isolated compounds of Clausena lansium stem bark: anti-trichomonal, antidiabetic, anti-inflammatory, hepatoprotective and antioxidant effects. J. Ethnopharmacol. 2009, 122, 10-19. [CrossRef] [PubMed]

8. Prasad, K.N.; Xie, H.H.; Hao, J.; Yang, B.; Qiu, S.X.; Wei, X.Y.; Chen, F.; Jiang, Y.M. Antioxidant and anticancer activities of 8-hydroxypsoralen isolated from wampee (Clausena lansium, (Lour.) Skeels) peel. Food Chem. 2010, 118, 62-66. [CrossRef]

9. Shen, D.Y.; Kuo, P.C.; Huang, S.C.; Hwang, T.L.; Chen, Y.Y.; Shieh, P.C.; Ngan, N.T.; Thang, T.D.; Wu, T.S. Constituents from the leaves of Clausena lansium and their anti-inflammatory activity. J. Nat. Med. 2017, 71, 96-104. [CrossRef] [PubMed]

10. Chinese Academy of Sciences. Flora of China; Science Press: Beijing, China, 1997.

11. Wang, X.H.; Hua, M.; Chen, Y.G. Comparison of traits of eleven yellow- wampee cultivars in Hainan. South China Fruits 2011, 40, 38-41. 
12. Zhao, J.Y.; Nan, P.; Zhong, Y. Chemical composition of the essential oils of Clausena lansium from Hainan island, China. Z. Naturforsch C. 2004, 59, 153-156. [CrossRef] [PubMed]

13. Chokepraser, P.; Khotavivattana, S.; Oupadisskoon, C.; Huang, T.C.; Chen, H.H. Volatile components of wampee fruits [Clausena lansium (Lour.) Skeels] treated by different drying conditions. Thammasat Int. J. Sci. Technol. 2006, 11, 66-71.

14. Pino, J.A.; Marbot, R.; Fuentes, V. Aromatic plants from western Cuba IV. Composition of the leaf oils of Clausena lansium (Lour.) Skeels and Swinglea glutinosa (Blanco) Merr. J. Essent. Oil Res. 2006, 18, 139-141. [CrossRef]

15. Silva, S.; Negri, M.; Henriques, M.; Oliveira, R.; Williams, D.W.; Azeredo, J. Candida glabrata, Candida parapsilosis and Candida tropicalis: biology, epidemiology, pathogenicity and antifungal resistance. FEMS Microbiol Rev. 2012, 36, 288-305. [CrossRef] [PubMed]

16. Wang, H.M.; Xu, J.P.; Guo, H.; Wu, J.Y.; Yi, G.H.; Pei, H.; Niu, L.N.; Li, Y. Patterns of Human Oral Yeast Species Distribution on Hainan Island in China. Mycopathologia 2013, 176, 359-368. [CrossRef]

17. Božović, M.; Garzoli, S.; Sabatino, M.; Pepi, F.; Baldisserotto, A.; Andreotti, E.; Romagnoli, C.; Mai, A.; Manfredini, S.; Ragno, R. Essential Oil Extraction, Chemical Analysis and Anti-Candida Activity of Calamintha nepeta (L.) Savi subsp. glandulosa (Req.) Ball-New Approaches. Molecules 2017, 22, 203-215.

18. Huang, Y.F.; Zhang, Y.M.; Huang, J.W.; Li, R.Z.; Liu, J. Study on chemical constituents of volatile oil and trace elements from fruits of Clausena lansium. China J. Chin. Mater. Med. 2006, 31, 898-900.

19. Chokeprasert, P.; Charles, A.L.; Sue, K.H. Volatile components of the leaves, fruits and seeds of wampee (Clausena lansium (Lour.) Skeels). J. Food Compos. Anal. 2007, 20, 52-56. [CrossRef]

20. Białoń, M.; Krzyśko-upicka, T.; Koszałkowska, M.; Wieczorek, P.P. The Influence of Chemical Composition of Commercial Lemon Essential Oils on the Growth of Candida Strains. Mycopathologia 2014, 177, $29-39$. [CrossRef] [PubMed]

21. Xu, S.C.; Zhou, H.L.; Liao, Y.Y.; Yang, R.; Yang, J.S. Extract Active Substances in Different Organ of Clausena lansium and the Activity to Colletotrchum musae. Food Res. Dev. 2010, 31, 65-68.

22. Perigo, C.V.; Torres, R.B.; Bernacci, L.C.; Guimarães, E.F.; Haberc, L.L.; Facanali, R.; Vieira, M.A.R.; Quecini, V.; Marques, M.O.M. The chemical composition and antibacterial activity of eleven Piper species from distinct rainforest areas in Southeastern Brazil. Ind. Crop. Prod. 2016, 94, 528-539. [CrossRef]

23. Chehregani, A.; Mohsenzadeh, F.; Mirazi, F.; Hajisadeghian, S.; Baghali, Z. Chemical composition and antibacterial activity of essential oils of Tripleurospermum disciforme in three developmental stages. Pharm. Biol. 2010, 48, 1280-1284. [CrossRef]

24. Soares, E.R.; Silva, F.M.A.; Almeida, R.A.; Lima, B.R.; Koolen, H.H.F.; Lourenço, C.C.; Salvador, M.J.; Flach, A.; Costa, L.A.M.A.; Souza, A.Q.L.; et al. Chemical composition and antimicrobial evaluation of the essential oils of Bocageopsis pleiosperma Maas. Nat Prod. Res. 2015, 29, 1285-1288. [CrossRef]

25. CLSI. Method for Antifungal Disk Diffusion Susceptibility Testing of Yeasts, 3rd ed.; Clinical and Laboratory Standards Institute: Wayne, PA, USA, 2009.

Sample Availability: Samples of the Clausena lansium EOs are available from the authors.

(C) 2019 by the authors. Licensee MDPI, Basel, Switzerland. This article is an open access article distributed under the terms and conditions of the Creative Commons Attribution (CC BY) license (http:/ / creativecommons.org/licenses/by/4.0/). 